\title{
Video Article \\ Analyzing the Photo-oxidation of 2-propanol at Indoor Air Level Concentrations Using Field Asymmetric Ion Mobility Spectrometry
}

\author{
Christopher P. Ireland ${ }^{1}$, Michael Coto ${ }^{1}$, Lauren Brown ${ }^{2}$, Russell Paris ${ }^{2}$, Caterina Ducati ${ }^{1}$ \\ ${ }^{1}$ Department of Materials Science and Metallurgy, University of Cambridge \\ ${ }^{2}$ Owlstone Nanotechnology
}

Correspondence to: Christopher P. Ireland at christopher.ireland@epfl.ch

URL: https://www.jove.com/video/54209

DOI: doi: $10.3791 / 54209$

Keywords: Chemistry, Issue 136, Photocatalysis, Indoor air purification, FAIMS, VOC degradation

Date Published: 6/14/2018

Citation: Ireland, C.P., Coto, M., Brown, L., Paris, R., Ducati, C. Analyzing the Photo-oxidation of 2-propanol at Indoor Air Level Concentrations Using Field Asymmetric Ion Mobility Spectrometry. J. Vis. Exp. (136), e54209, doi:10.3791/54209 (2018).

\section{Abstract}

We demonstrate a versatile protocol to be used for determining the effectiveness of photocatalysts in degrading indoor air concentration (ppb) volatile organic carbons (VOCs), illustrating this with a titanium dioxide based catalyst, and the VOC 2-propanol. The protocol takes advantage of field asymmetric ion mobility spectroscopy (FAIMS), an analysis tool that is capable of continuously identifying and monitoring the concentration of VOCs such as 2-propanol and acetone at the ppb level. The continuous nature of FAIMS allows detailed kinetic analysis, and long-term reactions, offering a significant advantage over gas chromatography, a batch process traditionally used in air purification characterization. The use of FAIMS in photocatalytic air purification has only recently been used for the first time, and with the protocol illustrated here, the flexibility in allowing alternative VOCs and photocatalysts to be tested using comparable protocols offers a unique system to elucidate photocatalytic air purification reactions at low concentrations.

\section{Video Link}

The video component of this article can be found at https://www.jove.com/video/54209/

\section{Introduction}

The quality of indoor air has come to the forefront recently. Perhaps surprisingly, indoor air contains a greater number of volatile organic carbons (VOCs), and in higher concentrations than outdoor air. ${ }^{1}$ With people spending over $80 \%$ of their time indoors, in places such as residential homes, workplaces, and transport including cars, trains, and aircraft, air quality can be a real issue. Many of the VOCs common in indoor air are mutagenic or carcinogenic, ${ }^{2,3}$ and so the removal of these is a key priority, especially as the phenomena of 'sick building syndrome' can lead to ill health and lost production through time off work. ${ }^{1}$ Air purification devices can include a photocatalyst, where a semiconductor, invariably titanium dioxide $\left(\mathrm{TiO}_{2}\right)$, activated with UV light, degrades the VOC through a photo-oxidation process. Photocatalysis is a growing area of research, with applications in water splitting for hydrogen production and pollutant degradation ${ }^{4,5,6,7}$; air purification is a particularly active area due to the commercial viability of this application ${ }^{8}$. However, detecting VOCs at concentrations that are present in indoor air (typically ppb) is challenging. With the kinetics of the photocatalytic reaction following Langmuir Hinshelwood kinetics ${ }^{9}$, the effectiveness of the photocatalyst at degrading VOCs at high concentrations is not representative of its effectiveness at low concentrations. Here we describe a versatile system and protocol for determining the effectiveness of photocatalysts at degrading VOCs at such low concentrations using field asymmetric ion mobility spectroscopy (FAIMS), illustrating this with a $\mathrm{TiO}_{2}$ based photocatalyst, and the model VOC 2-propanol.

Ionizing a gas flow, FAIMS separates and identifies chemical ions based on their mobility under a varying electric field at atmospheric pressure ${ }^{10,11,12}$. Molecules with a high proton affinity, such as VOCs are well suited to be separated and detected by FAIMS, with parts per billion $(\mathrm{ppb})$ resolution, and at ppb concentrations ${ }^{13}$. Capable of continuously monitoring multiple VOCs simultaneously, it is an ideal analysis to use in photocatalytic air purification testing, as in addition to monitoring the VOC used as pollutant. FAIMS can also detect intermediates or other VOC products with a high proton affinity from the photocatalytic reaction, a key requirement in proving that the photocatalyst is effective, as if the degradation is incomplete, some of the VOCs produced may be as toxic or more toxic than the VOC being degraded.

FAIMS has only recently been used for the first time in photocatalytic air purification applications ${ }^{14}$, and although not suggesting FAIMS is superior to gas chromatography, it clearly offers a versatile alternative, which has the potential to be a potent tool in studying air purification. Here we illustrate this technique with a protocol involving the photo-oxidation of 2-propanol with a titanium dioxide based photocatalyst. To generate 2propanol at the indoor air level concentrations permeation tubes are used ${ }^{15}$. Consisting of a PTFE tube containing the liquid VOC, that is sealed and crimped at both ends, under a constant flow, the VOC contained within the sealed PTFE permeation tube diffuses out at a constant rate, at concentrations comparable to indoor air. This flow is then passed into a reaction chamber containing the felt, and then into the FAIMS analyzer, where the identity and quantification of the VOC can be determined. FAIMS allows the concentration of 2-propanol to be determined, and via a library of spectra of know VOCs, the identity of additional VOCs produced during the photo reaction such as acetone determined through 
comparison of their spectra with the library. A key advantage of this technique is its flexibility: by simply changing the permeation tube or catalyst, alternative VOCs and catalysts can be tested.

\section{Makeup of VOC permeation tubes, and determination of its diffusion rate}

\section{Makeup of 2-propanol permeation tubes}

Note: To avoid contamination, wear gloves during this process.

Caution: 2-propanol is flammable and an irritant. Carry out this procedure away from any open flames. Wear gloves when handling 2propanol. Consult MSDS of 2-propanol for further information.

1. Measure out and cut a $14 \mathrm{~cm}$ length of PTFE tubing.

2. Seal and crimp one end of the tube by inserting a $2 \mathrm{~cm}$ length of PTFE rod into the end of the PTFE tubing, and then covering with a 2 $\mathrm{cm}$ metallic crimp

3. Place the PTFE tubing, rod and crimp into the crimping tool, and then place this into a vice. Turn the vice, tightening as much as possible to seal the PTFE tube with the crimp.

4. Pipette into the open-end of the PTFE tube an amount of 2-propanol, such that the PTFE tubing is around 1/3 full (approximately $3-4$ $\mathrm{mL})$.

5. Repeat 1.1.2 - 1.1.3 to seal and crimp the open end of the permeation tube; the permeation source is then complete.

\section{Determination of diffusion rate of VOC in the permeation tube}

1. Weigh the permeation tube, using a calibrated balance, to at least 4 decimal places, noting both the weight and time.

2. From a compressed air supply (ideally medical grade compressed air or equivalent), connect tubing (PTFE tubing, diameter $1 / 8$ in, internal diameter $0.063 \mathrm{in}$ ) to an in line pressure regulator. From the regulator, connect, using the same diameter PTFE tubing, to one of the ports of a GL45 4 port connector, screwed to a $250 \mathrm{~mL} \mathrm{GL} 45$ glass bottle. Block off two of the ports, and connect a length of PTFE tubing to the final port and guide this outlet to a fume hood.

3. Position the permeation tube into the GL45 glass bottle, and ensure there is a constant steam of compressed air at a flow rate of $2.5 \mathrm{~L}$ $\mathrm{min}^{-1}$. Alternatively, position the tube in the dilution chamber of the system as illustrated in Figure 1, and described in section 2.1 .

4. At specific time intervals (e.g. daily) repeat the weight measurement (1.2.1) and place back into the system (1.2.2). If the decrease in weight is undetectable using the balance, increase the time interval between weighing the permeation tube (e.g. weekly, bi-weekly). Note that this calibration process, depending on the diffusion rate, could take a time period of a few months.

5. Graph the diffusion rate with time in minutes on the $x$ axis and the mass loss in nanograms $(n g)$ on the $y$ axis. Draw a straight line between points; using the straight-line equation $(y=m x+c)$, determine the slope $(m)$ of the line. This is the permeation rate in $n g$ min $^{-1}$

\section{Photo-oxidation Reaction}

1. Set-up of equipment for use in the blank and photo-oxidation reaction (Figure 1)

1. Connect tubing (PTFE tubing, diameter $1 / 8$ in, internal diameter $0.063 \mathrm{in}$ ) from a compressed air supply to an in line pressure regulator. From this, connect a moisture trap, to ensure a consistent low level of moisture enters the setup. From here, connect the PTFE tubing to a scrubber to further clean the compressed air.

2. From the moisture trap or scrubber, connect, using the same diameter PTFE tubing, to a glass bottle, which will be the dilution chamber that will be used to hold the permeation tubes (GL45, $500 \mathrm{~mL}$ ). To ensure a gas tight connection, use a screw cap HPLC, GL45 4 port connector, complete with silicone seals: block off two of the ports, and connect the tubing from the scrubber or moister trap to one of the other two ports, ensuring the connection is tight. Screw HPLC GL 45 screw cap onto the $500 \mathrm{~mL}$ glass bottle.

3. Connect PTFE tubing to the final port or the HPCL GL45 screw cap, and then connect this to a second HPLC GL45 4 port connector. As with 2.1.2, block off two of the ports. Screw this HPLC FG45 screw cap on to a glass bottle (GL $45,250 \mathrm{~mL}$ ), which will be used as the reaction chamber.

4. Connect PTFE tubing to the final port on the HPLC GL45 screw cap, and from this, connect the tubing to the FAIMS gas analyzer, using Swagelok $1 / 8$ gas tight fittings. Ensure the external port of the gas analyzer is guided to a fume hood to ensure no contamination enters the laboratory work area.

5. Position the reaction chamber so that the center of the chamber is $15 \mathrm{~cm}$ from a UV lamp (e.g. a UV lamp, consisting of $2 \times 8 \mathrm{~W}$ tube lamps, with a peak photon emission wavelength of $356 \mathrm{~nm}$ ).

Caution: UV light is dangerous to eyes; ensure the lamp and reactor is surrounded by a metallic shield to avoid exposure to the light.

2. Photo-oxidation of 2-propanol

1. Place two 2-propanol permeation tubes assembled previously (1.1) in the dilution chamber of the setup described above. Place the catalyst (e.g., a titanium dioxide based felt, dimensions $55 \mathrm{~mm} \times 25 \mathrm{~mm} \times 1 \mathrm{~mm}$ ) in the reaction chamber, and ensure the catalyst is facing the UV lamp. Turn on the flow of compressed air, and adjust so the flow is $2.5 \mathrm{~L} \mathrm{~min}^{-1}$, and the pressure is 1 bar.

2. Turn on the FAIMS instrument, and set up the instrument so that the ion current of the 2-propanol can be seen. Using software configured for the FAIMS device, increase the RF waveform, so that distinct ion peaks can be seen on the spectrum being produced by the FAIMS instrument.

3. Using the software configured for the FAIMS device, monitor and record the ion current that is emanating from the distinct ion peaks seen on the spectrum produced by the FAIMS for a period of time, with the catalyst in the dark. The peaks will be 2-propanol, and water. At a set point (e.g. after leaving overnight), turn on the UV lamp, and monitor the FAIMS spectrum for the 2-propanol and water ion currents, plus additional signals from intermediate VOCs such as acetone. Using system software, increase or decrease the RF waveform to determine new signals emanating from the intermediate ions. 
Caution: Ensure both the UV light and reactor are covered with a metallic shield before the lamp is illuminated, and that the shield is present throughout the entire UV light reaction.

4. At a set point (e.g. after 4 hours), turn off the UV lamp, and continue to monitor the FAIMS spectrum for 2-propanol and additional peaks.

\section{Representative Results}

The FAIMS gas analyzer continuously produces spectra of ion current vs. compensation voltage during the course of the photo oxidation reaction described in 2.2, utilizing two 2-propanol permeation tubes in the dilution chamber, and a titanium dioxide based felt photocatalyst in the reaction chamber. Spectra typically produced by the FAIMS analyzer when the felt is in the dark, and when the felt is illuminated are illustrated in Figure 2a. To obtain the spectra with the FAIMS instrument, the RF waveform on the instrument is set to $64 \%$ of the maximum. At this RF waveform value, hydronium ions (water clusters), acetone monomers, and 2-propanol monomers which can be formed from the FAIMS instrument ionization process reach the detector in the FAIMS at distinct compensation voltages (cv), and so are separated on the spectra. Flowing individual gasses exclusively through the FAIMS system can be used to determine the spectra and compensation values for each gas ${ }^{16}$. On the spectrum, the peak at a compensation voltage of $-2.15 \mathrm{~V}$ is the hydronium ion, a water cluster ion formed when moisture in the air is ionized. The peak at a cv of $-0.14 \mathrm{~V}$ is that of 2-propanol ${ }^{14}$. The ion current is directly proportional to that of the 2-propanol concentration, and so using the diffusion rate (1.2), the concentration of 2-propanol entering the FAIMS can be determined. Similarly with acetone, occurring at a $\mathrm{cv}$ of $-1.44 \mathrm{~V}$. Figure $2 \mathrm{~b}$ shows the ion current measured at the specific peaks identified as 2-propanol and acetone in the spectra with the RF waveform at $64 \%$ of maximum, as a function of time throughout the photo-oxidation protocol described in section 2.2 . As subtle changes in flow and humidity can have an effect of shifting the ion current peak cV value positively or negatively, the peak height at a CV value of $\pm 0.2 \mathrm{~V}$ is used.

The amount of 2-propanol entering the FAIMS analyzer, with the reaction chamber in the dark increases over time. As 2-propanol enters the dilution chamber, 2-propanol is adsorbed onto the surface of the catalyst, which accounts for the initial low amount of 2-propanol entering the FAIMS. As time continues a higher ion current is recorded, indicating that a higher amount of 2-propanol enters the FAIMS. This suggests that the surface of the felt is being covered with 2-propanol, hence adsorption onto the catalyst is decreasing.

When the reactor chamber is illuminated, there is an immediate increase in 2-propanol entering the FAIMS. This implies that an amount of 2propanol desorbs from the surface of the felt, and enters the FAIMS analyzer. Concurrently, there is an increase in ion current from the peak at cv $-1.44 \mathrm{~V}$, which has previously been identified as acetone, indicating the felt under illumination has photo-oxidized 2-propanol to acetone. As time continues, the amount of 2-propanol decreases to a level significantly below the level at the initial point of illumination, and acetone continues to be detected, with both ion currents consistent over a period of around 3 hours. This implies that 2-propanol is consistently being photo-oxidized either to acetone, or to carbon dioxide and water. 2-propanol adsorbs onto the surface, is photo-oxidized, and the products desorb and enter the FAIMS, where acetone is recorded. After the light is turned off, the 2-propanol ion current increases, whilst the acetone ion current decreases implying the photo-oxidation has ceased.

The results are representative of the concentration of 2-propanol and acetone, continuously monitored at ppb concentrations. By comparing the steady state 2-propanol current under illumination with that of 2-propanol current entering the FAIMS before illumination, the effectiveness of the catalyst can be seen, with a greater decrease in 2-propanol entering the FAIMS indicative of a superior photocatalyst. The monitoring of additional VOCs also allows a better assessment of the effectiveness of the photocatalyst. In air purification applications, ideally the VOC should be degraded to carbon dioxide and water. Additional compounds detected demonstrate an ineffective catalyst or poor air purification strategy (flow rates, light intensity, humidity levels). FAIMS can monitor the photo-reaction, and so demonstrate the effectiveness of catalyst and air purification setup.

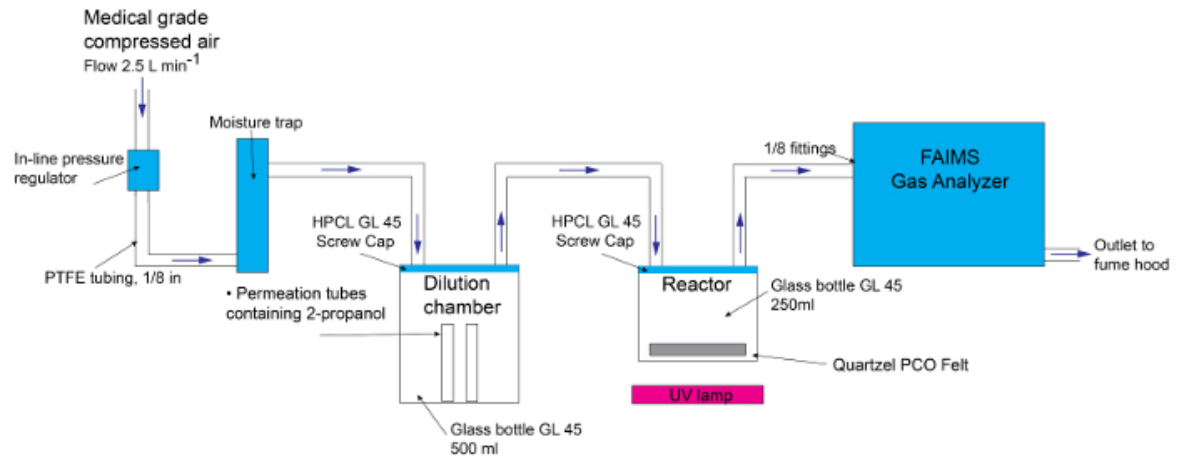

Figure 1. The Reactor Setup. Diagram illustrating the photocatalysis setup developed for use with the FAIMS gas analyzer (see 2.1). Please click here to view a larger version of this figure. 
(a)

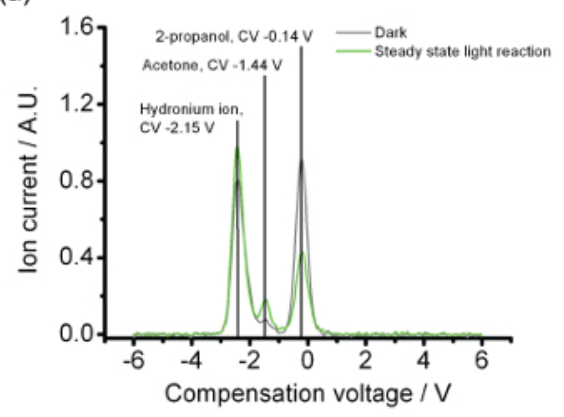

(b)

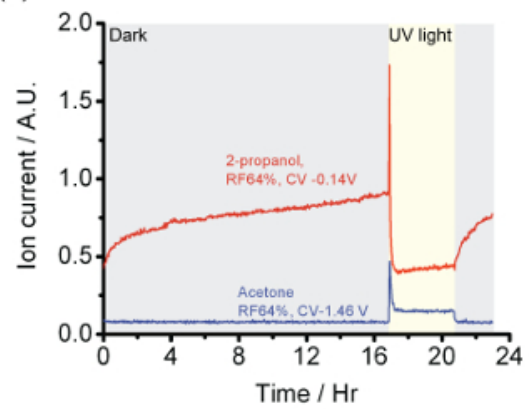

Figure 2. Typical Results. (a) Typical spectra produced by the FAIMS when the RF waveform is $64 \%$ of maximum when the reaction containing the felt is in the dark (grey line) and when it is illuminated (green line). (b) Graph showing the ion current at peaks from the compensation voltage vs ion current spectra produced during the 2-propanol photo-oxidation reaction when the RF waveform is at $64 \%$ of maximum; 2-propanol (red line) and acetone (blue line) shown, with the illuminated reaction highlighted. Please click here to view a larger version of this figure.

\section{Discussion}

The protocol describes an effective way of determining the effectiveness of the titanium oxide based catalyst, by determining its behavior in degrading a model VOC, 2-propanol, under UV illumination. Using FAIMS, the amount of 2-propanol can be monitored continuously throughout the reaction, in addition to any other VOC products that could be produced in the reaction, at concentrations comparable to indoor air. This continuous nature differs significantly from gas chromatography, traditionally used to monitor photocatalytic indoor air purification, which uses a batch process. An expensive, sensitive GC/MS system is generally required to determine the concentration of VOCs at such low concentrations, and detailed analysis of the photo-oxidation products generally requires further processing of the photo-oxidation products, such as adsorbing products onto activated carbon, and then desorbing them into the mass spectrometer. Whilst mass spectrometry is able to detect all products, a limitation of FAIMS is that only products with a high proton affinity can be detected. FAIMS is excellent at determining low concentration VOCs, but can be saturated at higher concentrations, which limits the system to indoor air level concentration applications. The advantages of FAIMS makes the system described here an effective, simple tool that can provide insights into photocatalytic reactions that gas chromatography is limited in achieving.

With the FAIMS system described here, medical grade air is used as the flow gas. With the FAIMS system being so sensitive, a high quality grade of air is critical in allowing the photo-oxidation to be analyzed. This ensures that any products detected are from the photo-oxidation process. Similarly, it is critical to ensure there is no leakage into the system, as laboratory air generally contains VOCs at concentrations the FAIMS is capable of detecting. The consumables listed for the setup of the system provide a reliable system, and continuous monitoring over a period of days has indicated no detectable VOCs when no catalyst or permeation tube is present.

Whilst the system is simple, it is also very flexible - alternative VOCs can be tested in this way, by simply making a permeation tub containing the alternative VOC, such as ethanol, acetone, or toluene, and following the protocol. Photocatalytic reactions are often affected by humidity. The system developed here operates under low humidity; however testing can be carried out at higher humidities buy introducing a humidifier into the system. Depending on the VOC used, it can result in the sensitivity of the FAIMS being reduced, but effective testing can be carried out. ${ }^{16}$

The continuous nature of FAIMS highlights an advantage over gas chromatography, which is traditionally used to determine the photocatalyst effectiveness in purifying air. ${ }^{16,17}$ Gas chromatography uses a batch process to collect and analyze air samples; FAIMS, with its continuous nature, allows a more detailed look at the kinetics of the photocatalytic reaction, which can be challenging to interpret with the batch gas chromatography technique. The simplicity of FAIMS is another advantage. In order to carry out the complex analysis of multiple VOCs FAIMS is capable of, the gas chromatograph will need to be linked to a mass spectrometer, which can be expensive and require additional processing. Additionally, to carry out long term reactions with a gas chromatograph, an expensive automated system would be required, or labor intensive sampling; this is not the case with FAIMS.

The continuous nature of FAIMS offers significant advantages over gas chromatography that can be utilized to gain a greater understanding of the photocatalysis process at these ppb concentrations. Moreover, the simple setup illustrated here is flexible, allowing alternative photocatalysts and VOCs to be tested under comparable conditions, further improving the understanding of the photocatalytic process.

\section{Disclosures}

The authors Lauren Brown and Russell Paris are employees of Owlstone Nanotechnology, the company that manufactures the FAIMS analysis instrument that is used in this article.

\section{Acknowledgements}

The authors are grateful for the financial support from the ERC, under grant number 259619 PHOTO EM and grant number 620298 PHOTO AIR (Proof of Concept). 


\section{References}

1. Wang, S. B., Ang, H. M., \& Tade, M. O. Volatile organic compounds in indoor environment and photocatalytic oxidation: State of the art. Environ. Int. 33 (5), 694-705 (2007).

2. Shah, J. J., \& Singh, H. B. Distribution of Volatile Organic-Chemicals in Outdoor and Indoor Air - a National Vocs Data-Base. Environ. Sci. Technol. 22 (12), 1381-1388 (1988).

3. Jones, A. P. Indoor air quality and health. Atmos. Environ. 33 (28), 4535-4564 (1999).

4. Hoffmann, M. R., Martin, S. T., Choi, W. Y., \& Bahnemann, D. W. Environmental Applications of Semiconductor Photocatalysis. Chem. Rev. 95 (1), 69-96 (1995).

5. Mills, A., \& LeHunte, S. An overview of semiconductor photocatalysis. J. Photochem. Photobiol., A. 108 (1), 1-35 (1997).

6. Osterloh, F. E. Inorganic materials as catalysts for photochemical splitting of water. Chem. Mater. 20 (1), 35-54 (2008).

7. Osterloh, F. E. Inorganic nanostructures for photoelectrochemical and photocatalytic water splitting. Chem. Soc. Rev. 42 (6), $2294-2320$ (2013).

8. Paz, Y. Application of $\mathrm{TiO}_{2}$ photocatalysis for air treatment: Patents' overview. Appl. Catal., B. 99 (3-4), $448-460$ (2010).

9. Herrmann, J. M. Photocatalysis fundamentals revisited to avoid several misconceptions. Appl. Catal., B. 99 (3-4), $461-468$ (2010).

10. Guevremont, R. High-field asymmetric waveform ion mobility spectrometry: A new tool for mass spectrometry. J. Chromatogr. A. 1058 (1-2), 3-19 (2004).

11. Kolakowski, B. M., \& Mester, Z. Review of applications of high-field asymmetric waveform ion mobility spectrometry (FAIMS) and differential mobility spectrometry (DMS). Analyst. 132 (9), 842-864 (2007).

12. Kanu, A. B., Dwivedi, P., Tam, M., Matz, L., \& Hill, H. H. Ion mobility-mass spectrometry. J. Mass Spectrom. 43 (1), 1-22 (2008).

13. Field Asymmetric lon Mobility Spectrometry. FAIMS. http://www.owlstonenanotech.com/faims (2015).

14. Ireland, C. P., \& Ducati, C. Investigating the photo-oxidation of model indoor air pollutants using field asymmetric ion mobility spectrometry. J. Photochem. Photobiol., A. 312 1-7 (2015).

15. Owlsteone Nanotech. Permeation Tubes and Diffusion Tubes. http://www.owlstonenanotech.com/calibration-gas-generator/permeation-tubesand-diffusion-tubes (2015).

16. Vildozo, D., Ferronato, C., Sleiman, M., \& Chovelon, J. M. Photocatalytic treatment of indoor air: Optimization of 2-propanol removal using a response surface methodology (RSM). Appl. Catal., B. 94 (3-4), 303-310 (2010).

17. Vildozo, D., Portela, R., Ferronato, C., \& Chovelon, J. M. Photocatalytic oxidation of 2-propanol/toluene binary mixtures at indoor air concentration levels. Appl. Catal., B. 107 (3-4), 347-354 (2011). 